This article was published as

Philosophical Transactions of the Royal Society A-Mathematical Physical and Engineering Sciences 379: 20200302 (2021)

DOI: https://doi.org/10.1098/rsta.2020.0302 


\title{
The role of thermal diffusion, particle clusters, hydrodynamic and magnetic forces on the flow behaviour of magneto-polymer composites
}

\author{
William R. Suarez-Fernandez, ${ }^{1,2}$,Juan DG Duran ${ }^{1,3}$, Modesto T Lopez-Lopez ${ }^{1,3, *}$ \\ ${ }^{1}$ Department of Applied Physics, University of Granada, 18071 Granada, Spain \\ ${ }^{2}$ Faculty of Engineering Sciences and Industries, Universidad UTE, 170129 Quito, Ecuador \\ ${ }^{3}$ Instituto de Investigación Biosanitaria IBS.Granada, 18012 Granada, Spain \\ WRS-F, 0000-0002-7211-773X; \\ MTL-L, 0000-0002-9068-7795; \\ JDGD, 0000-0002-5586-1276;
}

Keywords: rheology; Péclet number; viscosity; viscoelasticity; polymer solution; magnetic particles

\section{Summary}

In this paper we study the shear-induced flow of magneto-polymer composites, consisting of dispersions of magnetic particles in solutions of polymers, as a competition between the colloidal forces amid particles and their bulk transport induced by the hydrodynamic forces. For this aim, we analyse the role of different experimental parameters. Firstly, by using only solutions of a well-known anionic polymer (sodium alginate) we provoke a moderate hindering of particle movement, but keeping the liquid-like state of the samples. On the contrary, a gel-like behaviour is conferred to the samples when a cationic polymer (chitosan) is additionally added, which further reduces the particle movement. We analyse the effect of an applied magnetic field, which opposes to particle transport by hydrodynamic forces, by inducing magnetic attraction between the particles. We perform the analysis under both stationary and oscillatory shear. We show that by using dimensionless numbers the differences between samples and experimental conditions are emphasized. In all cases, as expected, the transport of particles driven by bulk hydrodynamic forces dominates at high values of the shear rate.

\section{Introduction}

Hydrogels are three-dimensional (3-D) networks of polymer chains embedded by water or other biological fluids[1]. In hydrogels usually less than $10 \%$ of the mass is due to the polymer, water being the main component, resulting in a largely porous microstructure, resembling the natural extracellular matrix of living tissues. Because of this resemblance, hydrogels have been the focus of intense research in the last few decades, aiming to products with potential applications in biomedicine and other fields [2,3]. From the viewpoint of the bonding between polymer chains, hydrogels can be classified into chemical hydrogels and physical hydrogels. Covalent bonding is the mechanism behind the 3-D network in the former, whereas physical interactions, such as electrostatic attraction, hydrogen bonding, or even entanglement between polymer chains, are the mechanisms maintaining the polymer structure in the latter [4]. Chemical hydrogels 
are stronger than physical hydrogels, however, self-healing is much more common presented in networks based in physical interactions than in chemical bonding, which represents a major advantage of physical hydrogels. Physical interactions between polymer chains are also present in other systems consisting of polymer and water, e.g., polymer solutions, that do not meet the requirements for being classified as hydrogels. For this, different subjective criteria are found in literature, such as self-supporting ability, or solid-like appearance. On the contrary, from the rheological viewpoint, a clear criterium is stablished for hydrogels: that their storage modulus $\left(G^{\prime}\right)$ is higher than the loss modulus $\left(G^{\prime \prime}\right)$ in mechanical spectra $[5,6]$. Polymer networks can be combined with inorganic particles to provide the hydrogel with smart properties [7]. We refer to these materials as polymer composites, and they can present the interesting property of being responsive to external stimuli. An example are magneto-polymer composites that are obtained by a combination of polymer networks and magnetic particles in a continuous medium[8]. Magneto-polymer composites are attracting great interest because they combine the magnetic character of particles and the deformability of polymer networks. Consequently, they can respond to external magnetic stimulus by motion, deformation or changes in the mechanical properties[9,10]. Interestingly, remote magnetic fields represent one of the most attractive ways of actuation due to the ease of use, prompt response and safe penetration in biological environments.

In a recent article, we investigated magneto-polymer composites consisting of micron-sized iron particles dispersed in solutions of two different alginate polymers (with medium and high molecular weight) [11]. We found that magneto-polymer composites based on alginate polymer of medium molecular weight, behaved as liquid-like materials $\left(G^{\prime}<G^{\prime \prime}\right)$ in the absence of applied magnetic field, no matter polymer concentration. On the contrary, for magneto-polymer composites based on alginate of high molecular weight, we found a critical polymer concentration above which the composites behaved as gel-like materials $\left(G^{\prime}>G^{\prime \prime}\right)$ as a consequence of polymer chain entanglement. In all cases, the application of a magnetic field resulted in gel-like behaviour due to the formation of particle structures induced by magnetic attraction between magnetized particles.

In the present work, we demonstrate that starting from a liquid-like solution of medium-molecular-weight alginate ( 15 weight $\%$ of polymer concentration), it is possible to obtain a gel-like behaviour by addition of a small amount of chitosan polymer (less than 1 weight \%) under mildly acidic medium. The reason behind this transformation from liquid-like to gel-like behaviour must be the electrostatic interaction between anionic alginate ions and cationic chitosan ions. Based on these polymer solutions we fabricated magnetopolymer composites by dispersing iron microparticles. We investigated thoroughly the rheological properties of these alginate-chitosan-iron composites. For comparison purposes, we use magneto-polymer composites consisting of suspensions of iron particles in solutions of alginate polymer. In our rheological analysis we focused on the role of thermal diffusion, particle clusters, hydrodynamic and magnetic forces on the resulting flow behaviour of magneto-polymer composites. For this aim we used dimensionless numbers. In particular, we used Péclet number $(\mathrm{Pe})$, which is a dimensionless number relevant in the study of transport phenomena in continuum media [12]. In the flow of suspensions of particles, $\mathrm{Pe}$ is defined as the ratio of the rate of transport of the particles by hydrodynamic forces to the rate of diffusion of the particles by thermal motion. For suspensions of magnetic particles, their magnetic field-induced assembly, which opposes both thermal motion and hydrodynamic transport, also plays a prominent role in the flow behaviour. To account for its role, we use the dimensionless lambda number $(\lambda)$, which is defined as the ratio between magnetostatic energy of attraction between particles and energy of thermal diffusion [13]. We show that the study based on these dimensionless numbers allows a better analysis of the differences between samples and experimental conditions.

\section{Materials and methods}

\section{(a) Materials}

As polymer materials we used sodium alginate (SA) from brown algae (CAS Number 9005-38-3, Sigma Aldrich, USA) and chitosan from carb shells (CAS Number 9012-76-4, Sigma Aldrich, USA). We also used acetic acid (96\% of purity) supplied by Panreac Química S.A. (Spain), and sodium hydroxide, supplied by Scharlau (Spain). As dispersion medium we used deionized water, and as magnetic phase Fe-HQ powder (BASF, Germany), consisting of spherical iron particles of diameter $0.9 \pm 0.3 \mu \mathrm{m}$, volumetric mass density $7.88 \pm 0.16 \mathrm{~g} \mathrm{~cm}^{-3}$, and saturation magnetization $\mathrm{M}_{\mathrm{S}}=1721 \pm 2 \mathrm{kA} / \mathrm{m}$. 
(b) Preparation of solutions of alginate polymer and solution of alginate-chitosan polymers

SA polymer was readily soluble in water without any further treatment. We dissolved it in deionized water at a concentration of 15 weight \%. We used mechanical mixing to accelerate the dissolution and homogenize the resulting alginate solutions. On the contrary, chitosan polymer was not soluble in water at neutral $\mathrm{pH}$, but it is in acetic acid solutions. Therefore, we proceeded as it follows for the preparation of mixed solutions of chitosan and alginate. First, we dissolved, by magnetic stirring for $1.5 \mathrm{~h}, 0.25 \mathrm{~g}$ of chitosan polymer in 25 $\mathrm{mL}$ of a $0.2 \mathrm{M}$ aqueous solution of acetic acid. The $\mathrm{pH}$ of this chitosan polymer solution was 3.8. Afterwards, we added $4 \mathrm{~mL}$ of a $1 \mathrm{M}$ aqueous solution of sodium hydroxide and placed the resulting mixture under magnetic stirring for $2.5 \mathrm{~h}$. Afterwards, the $\mathrm{pH}$ was 5.8 . Then, we added $5 \mathrm{~g}$ of SA polymer and used mechanical stirring for $20 \mathrm{~min}$ to accelerate the dissolution of this polymer.

(c) Preparation of suspensions of magnetic particles in polymer solutions

We prepared suspensions of magnetic particles, by dispersing HQ iron powders at a concentration of 5 vol.\% in the polymer solutions. We homogenised the resulting mixtures by mechanical mixing for $10 \mathrm{~min}$.

\section{(d) Rheological characterization of the solutions and suspensions}

For the characterisation of the rheological properties, we used a rotational (magneto)rheometer (Physica MCR 300) provided with a plate-plate geometry of $20 \mathrm{~mm}$ of diameter. We used the commercial magnetocell provided with the Physica MCR300 rheometer for the application of magnetic fields to the samples during measurements. We carried out all measurements at a constant temperature of $25 \pm 0.1{ }^{\circ} \mathrm{C}$. We subjected the samples to both, steady state and oscillatory tests. In the former, we subjected the samples to ramps of shear rate, $\dot{\gamma}$, of increasing value and the resulting shear stress, $\sigma$, and viscosity, $\eta$, were monitored. In oscillatory tests, we subjected the samples to oscillatory shear strains, monitoring the resulting oscillatory shear stress. From these oscillatory tests, we obtained the values of the storage $\left(G^{\prime}\right)$ and loss $\left(G^{\prime \prime}\right)$ moduli. We performed two different oscillatory tests: amplitude sweeps and frequency sweeps. In amplitude sweeps we subjected the samples to oscillatory shear of constant frequency $(1 \mathrm{~Hz})$ and increasing shear amplitude, whereas in frequency sweeps, we subjected the samples to oscillatory shear of constant amplitude and increasing frequency. For frequency sweeps we maintained strain amplitude values well into the linear viscoelastic region (LVR): $10 \%$ of strain for samples containing only alginate polymer and $0.1 \%$ of strain for samples containing both alginate and chitosan polymers.

Rheological results shown in this manuscript for each sample and experimental condition represent the mean and standard deviation of at least three separate measurements. In general, we excluded noise and outliers from the analysis.

\section{(e) Self-healing capacity of the samples}

For the analysis of the self-healing capacity of the samples we used a controlled-stress Haake Mars III rheometer, provided with concentric cylinder measuring system, of $16 \mathrm{~mm}$ of internal diameter and $17 \mathrm{~mm}$ of external diameter. We subjected the samples to an oscillatory shear strain of constant frequency $(1 \mathrm{~Hz})$ and constant amplitude of $0.1 \%$, and monitored the viscoelastic moduli as a function of time. At time $t \approx 100$ $\mathrm{s}$, and for a total duration of $60 \mathrm{~s}$, we stopped the oscillatory shear strain and subjected the samples to a shear rate of $1000 \mathrm{~s}^{-1}$, large enough to break the internal structure of the samples. Afterwards $(t \approx 160 \mathrm{~s})$, the shear rate was stopped, and the samples were subjected again to oscillatory shear strain of constant frequency (1 $\mathrm{Hz}$ ) and constant amplitude of $0.1 \%$, and monitored the viscoelastic moduli until $t \approx 1160 \mathrm{~s}$. 
(a)

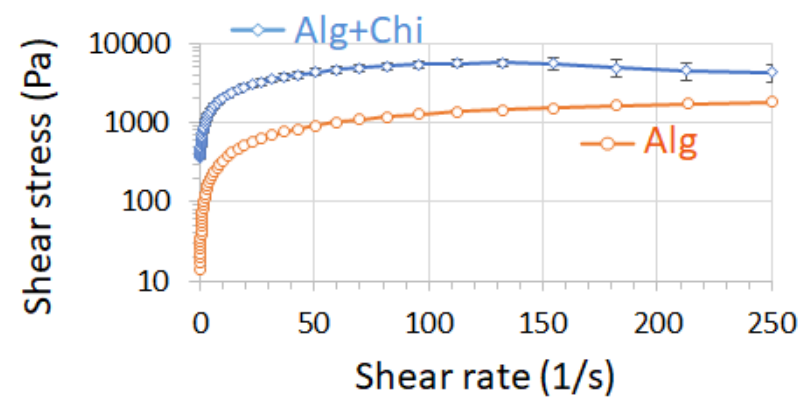

(c)

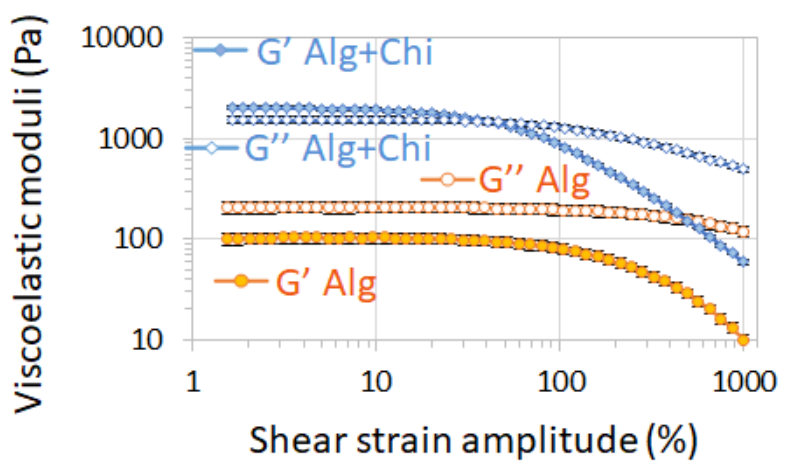

(b)

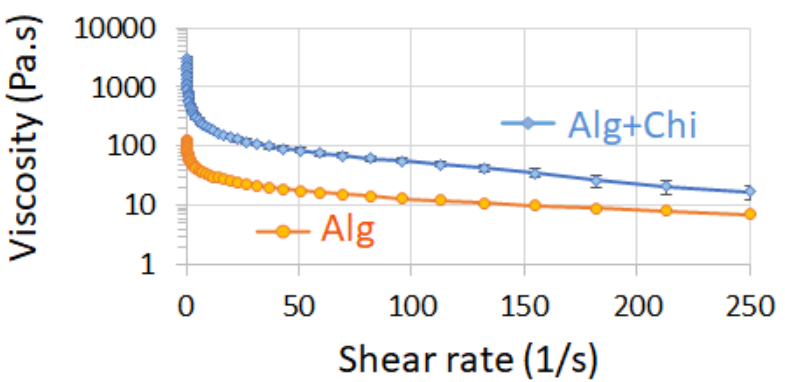

(d)

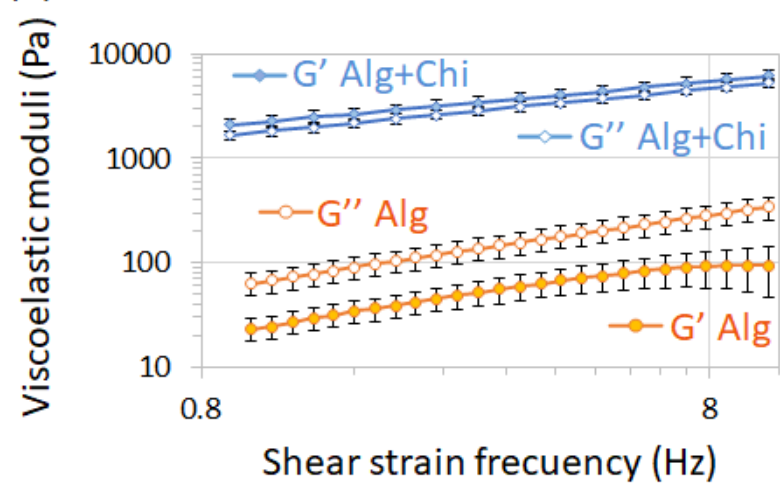

Figure 1. Rheological characterization of polymer solutions. (a) Rheograms: curves of shear stress vs. shear rate. (b) Viscosity vs. shear rate curves. (c) Amplitude sweeps: curves of storage (G') and loss $\left(G^{\prime \prime}\right)$ moduli vs. shear strain amplitude for a fixed frequency of $1 \mathrm{~Hz}$. (d) Frequency sweeps: curves of $\mathrm{G}^{\prime}$ and $G^{\prime \prime}$ vs. shear strain frequency for a fixed amplitude within the LVR. Alg: sodium alginate solution at 15 weight \%; Alg+Chi: polymer solution containing 15 weight $\%$ of sodium alginate and 0.75 weight \% of chitosan. Curves for Alg samples are taken from our previous work [11].

\section{Experimental results}

(a) Rheological characterization of polymer solutions

We first studied the rheological properties of polymer solutions (Figure 1). The steady state analysis (Figures $1 \mathrm{a}$ and $1 \mathrm{~b}$ ) demonstrated apparent yield stress and strong shear thinning behaviour, both phenomena more intense for solutions of alginate and chitosan (Alg+Chi sample) than for solutions of alginate (Alg sample). Both the apparent yield stress and the strong shear thinning behaviour are manifestations of the entanglement of the polymer chains that constitute the solution. In addition, sodium alginate has a pKa as low as 3 even in strong acidic media, and thus it can be expected that it will be dissociated into $\mathrm{Na}^{+}$and the negative alginate ion in the $\mathrm{Alg}+\mathrm{Chi}$ sample, whereas chitosan has an ample number of amino groups (pKa around 6.5) that tend to remain protonated at acidic and neutral $\mathrm{pH}$ [14]. As a result, in the mildly acidic conditions of the Alg+Chi sample, electrostatic attraction between alginate and chitosan molecules is expected. This fact justifies the one order of magnitude larger values of shear stress and viscosity for Alg+Chi sample with respect to Alg sample despite the similar total concentration of polymers. This electrostatic attraction between alginate and chitosan can be better induced from the analysis of the viscoelasticity of the samples (Figures 1c and 1d). As observed, Alg sample demonstrated the liquid-like behaviour typical of polymer solutions, characterized by values of the loss modulus $\left(G^{\prime \prime}\right)$ larger than those of the storage modulus $\left(G^{\prime}\right)$ and a dependence of both moduli to increase linearly with frequency in a double logarithmic plot (Figure 1d) [15]. On the contrary, Alg+Chi sample demonstrated a behaviour typical of weak gel, with $\mathrm{G}^{\prime}$ slightly larger than $G^{\prime \prime}$ in the linear viscoelastic region (LVR), and a less pronounced increase of both moduli with frequency - note that LVR is defined from amplitude sweeps (Figure 1c) as the region of low values of the shear stress amplitude, for which $G^{\prime}$ and $G^{\prime \prime}$ remain approximately constant, before the sharp decay of $\mathrm{G}^{\prime}$ that marks the onset of the nonlinear viscoelastic region (NLVR). 
(a)

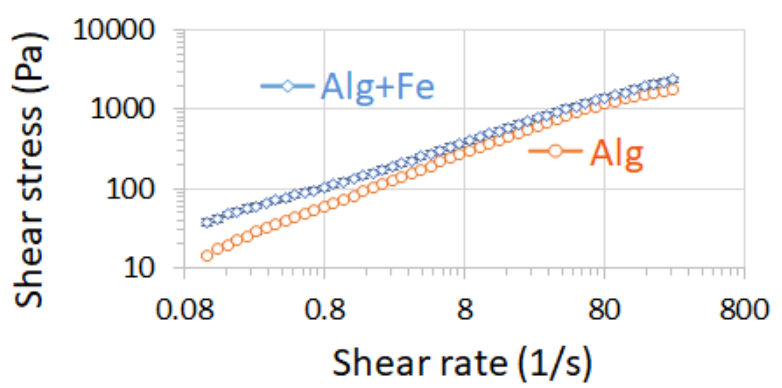

(c)

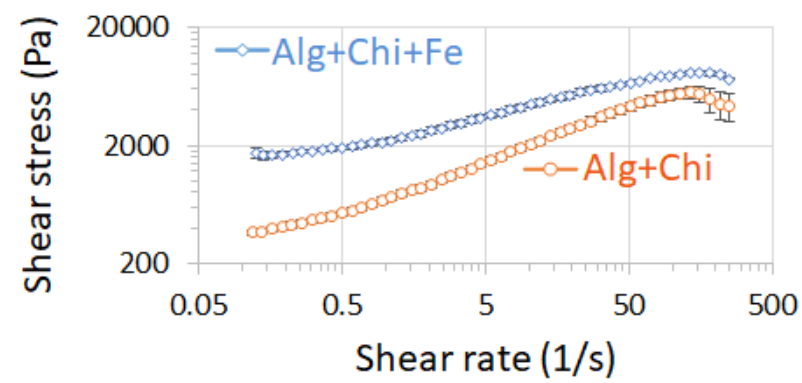

(b)

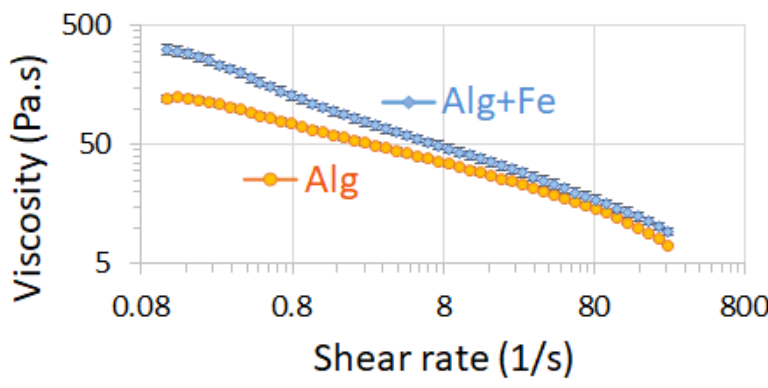

(d)

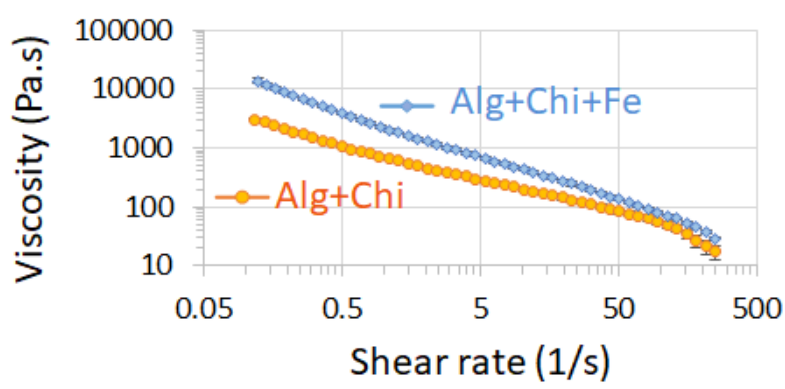

Figure 2. Effect of addition of iron particles on the steady state rheological behaviour. (a) and (c) Rheograms. (b) and (d) Viscosity vs. shear rate curves. Alg: sodium alginate solution at 15 weight \%; Alg+Chi: polymer solution containing 15 weight $\%$ of sodium alginate and 0.75 weight $\%$ of chitosan. Alg+Fe: suspension of iron particles at a concentration of 5 vol. \% in Alg solution; Alg+Chi+Fe: suspension of iron particles at a concentration of 5 vol. \% in Alg+Chi solution. Curves for Alg samples are taken from our previous work [11].

(b) Analysis of the stationary state measurements in suspensions of iron particles in polymer solutions

Let us first study the influence of addition of iron particles at a concentration of 5 vol. \% to the steady-state response of solutions of polymers (Figure 2). As observed, for both solutions of sodium alginate and solutions of sodium alginate and chitosan, the addition of iron particles resulted in the enhancement of the shear stress (Figures 2a and 2c) and the viscosity (Figures $2 b$ and $2 d$ ) for a given value of shear rate. In a previous work we demonstrated for magnetic alginate hydrogels that the inclusion of iron particles like these of the present work did not provoke changes at the microscopic level in the polymer arrangement of the alginate network [16]. What is more, in that previous work, we demonstrated that the enhancement of viscoelastic moduli of alginate hydrogels with particle content was well predicted by a modified KriegerDougherty (KD) equation, and thus, this enhancement was just due to the hydrodynamic interaction between particles (and their aggregates) with the dispersing medium. We will assume in this work that the same reasoning is held for the suspension under study. Keeping this in mind, in what follows we will analyse the role of thermal diffusion, hydrodynamic and magnetic forces on the flow behaviour of magneto-polymer composites. 
(a)

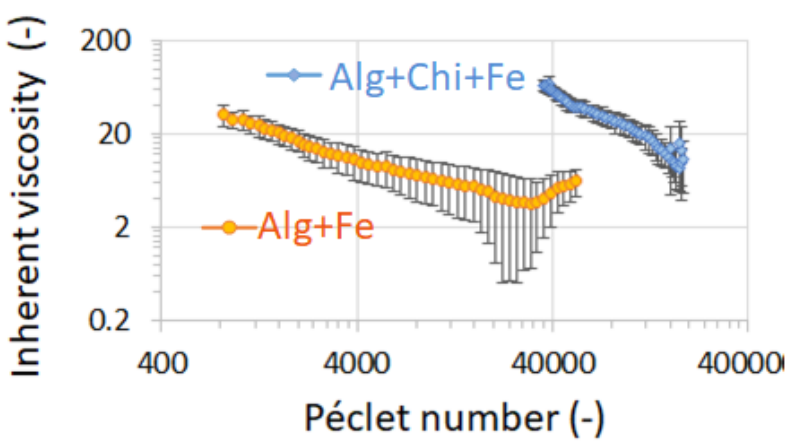

(b)

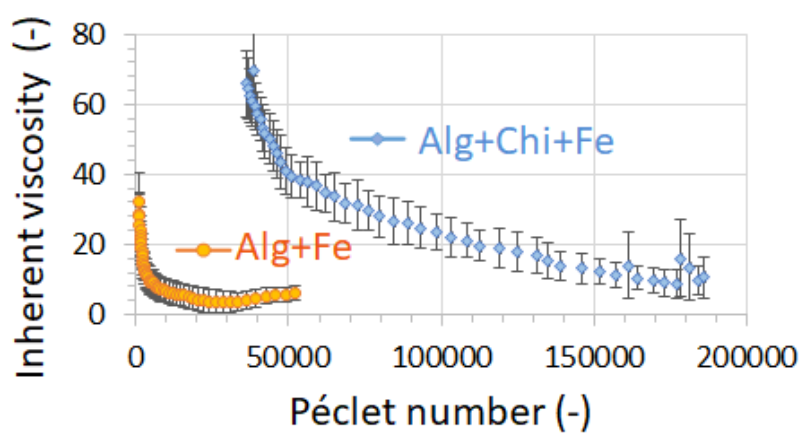

Figure 3. Inherent viscosity vs. Péclet number. (a) Double logarithmic scale; (b) Double linear scale. $\mathrm{Alg}+\mathrm{Fe}$ : suspension of iron particles at a concentration of $5 \mathrm{vol} . \%$ in sodium alginate solution at 15 weight $\%$; $\mathrm{Alg}+\mathrm{Chi}+\mathrm{Fe}$ : suspension of iron particles at a concentration of $5 \mathrm{vol} . \%$ in polymer solution containing 15 weight $\%$ of sodium alginate and 0.75 weight $\%$ of chitosan.

The use of dimensionless numbers in rheology is a common strategy to get a rigorous analysis of the differences between samples and experimental conditions, as well as to study the influence of different phenomena. An appropriate dimensionless number in transport phenomena in a continuum is Péclet number $(P e)$, that for a particle of radius $a$ in a medium of viscosity $\eta_{0}$ is defined as it follows [12]:

$$
P e=\frac{\eta_{0} a^{3} \dot{\gamma}}{k T}=\frac{\sigma a^{3}}{k T},
$$

with $k$ being the Boltzmann constant and $T$ the absolute temperature. From its definition, it is clear that $P e$ accounts for the ratio between hydrodynamic energy due to shear and the thermal energy $(k T)$. At low Pe numbers the effect of thermal motion influences the rheology of the suspensions because it leads to a disordered state in which flow is difficult (resulting in an increased viscosity), whereas as the hydrodynamic energy increases, the effect of thermal energy progressively disappears giving rise to ordinated particle structures that facilitate flow [12]. Therefore, a shear thinning behaviour is usually demonstrated in rheograms of particulate suspensions. To elucidate the role of particles in the viscosity, it seems appropriate to use the inherent viscosity, $\eta_{i n h^{\prime}}$, which is defined as it follows:

$$
\eta_{i n h} \equiv \frac{\eta-\eta_{0}}{\phi \eta_{0}},
$$

where $\eta_{0}$ is the viscosity of the dispersing medium (polymer solutions in our work) and $\phi$ is the particle volume fraction. By plotting the inherent viscosity as a function of Péclet number we may analyse the interplay between particle diffusion and hydrodynamic energy due to shear (Figure 3). As observed, for both suspensions, the effect of thermal diffusion seems to be negligible in view of the very large values of Péclet number. Nevertheless, it is obvious from Figure 3 that a shear thinning behaviour take place. In the present work, and in view of the very large values of Péclet number, the shear thinning behaviour should result as an effect of the destruction of the large particle clusters that constitute the sample. At this point it is important to remark that for such kind of iron particles, the existence of large particle clusters was demonstrated in previous works [11]. Note also that the existence of shear thinning behaviour associated to the fracture of aggregates was also proved in previous works - see for example [17]. From Figure 3 it is also evident that transport phenomena are more prominent (much larger Péclet number) in the suspension of iron particles in the solution of sodium alginate and chitosan (sample Alg $+\mathrm{Chi}+\mathrm{Fe}$ ). On the contrary, for Alg+Fe sample, a faster and more pronounced decrease with Péclet number of the inherent viscosity, toward a plateau value, is observed (this phenomenon is better seen in linear scale - Figure 3b), evidencing the fast transition from a regime with aggregates of particles being dominant, to a regime completely governed by transport induced by hydrodynamic forces. This transition is also observed in the suspension of iron particles in the solution of sodium alginate and chitosan, but it is more gradual, which indicates a smaller influence of particle aggregates in a gel-like sample.

Let us now study the influence of an applied magnetic field on the rheograms. An adequate dimensionless parameter to quantify the strength of interparticle magnetostatic forces against energy of thermal motion is the $\lambda$ parameter defined as it follows [13]:

$$
\lambda \equiv \frac{\pi \mu_{0} \mu_{f} \beta^{2} a^{3} H^{2}}{2 k T},
$$

Phil. Trans. R. Soc. A. 
were $\mu_{0}$ and $\mu_{f}$ are respectively the magnetic permeability of vacuum and the relative magnetic permeability of the carrier, $\beta=\left(\mu_{p}-\mu_{f}\right) /\left(\mu_{p}+2 \mu_{f}\right)$ is the magnetic contrast factor, $\mu_{p}$ the relative magnetic permeability of the particles, and $H$ the strength of the applied magnetic field. For $\lambda$ values higher than 1 the magnetostatic forces dominate over thermal diffusion and formation of particle structures induced by the field are expected, provided that the elasticity of the carrier allows particle motion. For the particles and magnetic fields under study in the present work, orders of magnitude of $\lambda$ are given in Table 1 -note that $\beta \approx 1$ for ferromagnetic particles of large permeability in a diamagnetic carrier $\left(\mu_{f}=1\right)$, as it is the case of the present work, and accordingly, we considered $\beta=1$ in calculations of Table 1 . As observed, magnetostatic forces largely dominate over thermal diffusion and, thus, magnetic field-induced particle structuration is expected in the present work.

Table 1. Values of the applied magnetic field and orders of magnitude of $\lambda$ parameter - equation (3).

\begin{tabular}{|l|c|c|c|c|}
\hline $\begin{array}{l}\text { Magnetic field strength, } \\
H,(\mathrm{kA} / \mathrm{m})\end{array}$ & 73.5 & 156 & 229 & 281 \\
\hline$\lambda$ parameter $(-)$ & 200,000 & $1,000,000$ & $2,000,000$ & $3,000,000$ \\
\hline
\end{tabular}

A general view of the inherent viscosity vs. Péclet number for $\mathrm{Alg}+\mathrm{Fe}$ and $\mathrm{Alg}+\mathrm{Chi}+\mathrm{Fe}$ samples for all the applied field intensities under study is presented in Figure 4 - note that both samples where investigated under the same range of applied shear rates, demonstrating the adequacy of dimensionless numbers to differentiate between samples and experimental conditions. As observed, three main differential features are presented by $\mathrm{Alg}+\mathrm{Fe}$ and $\mathrm{Alg}+\mathrm{Chi}+\mathrm{Fe}$ samples. The former present more pronounced shear thinning behaviour than the latter. Furthermore, curves of $\mathrm{Alg}+\mathrm{Chi}+\mathrm{Fe}$ sample are in a region of larger Péclet number, evidencing again smaller influence of aggregates of particles on the rheological properties of the sample in all cases the very large Péclet numbers demonstrate a negligible effect of thermal diffusion. Finally, the effect of the applied magnetic field appears less intense in $\mathrm{Alg}+\mathrm{Chi}+\mathrm{Fe}$ sample, which should be also connected with the reduced mobility of particles in the gel-like medium that constitutes the Alg+Chi solution.

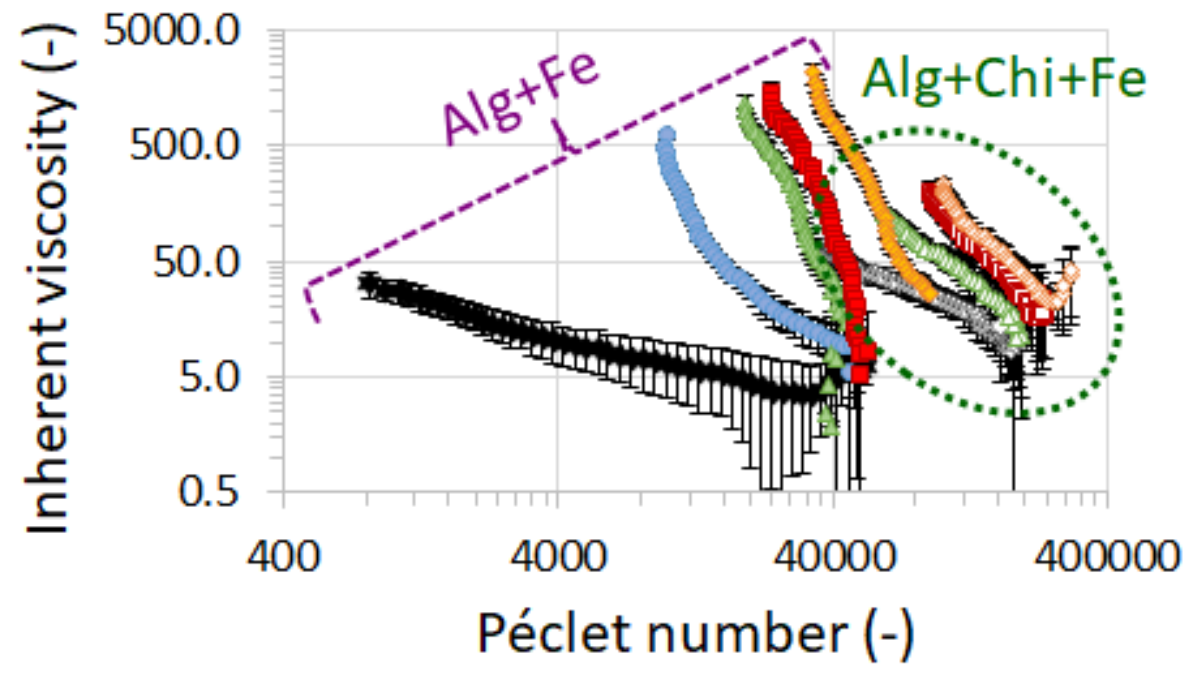

Figure 4. General view of the curves of inherent viscosity vs. Péclet number for different intensities of the applied magnetic field. Note that the curves are distributed in two groups: Alg+Fe: suspension of iron particles at a concentration of 5 vol. $\%$ in sodium alginate solution at 15 weight \%; Alg+Chi+Fe: suspension

of iron particles at a concentration of 5 vol. $\%$ in polymer solution containing 15 weight $\%$ of sodium

alginate and 0.75 weight $\%$ of chitosan. For each group, the intensity of the applied magnetic field, $H$, increases from the curve most to the left to the curve most to the right accordingly to the following values: Alg+Fe: $H=0 \mathrm{kA} / \mathrm{m} ; H=73.5 \mathrm{kA} / \mathrm{m} ; H=156 \mathrm{kA} / \mathrm{m} ; H=229 \mathrm{kA} / \mathrm{m} ; H=281 \mathrm{kA} / \mathrm{m} ;$ Alg+Chi+Fe: $H=0$

$\mathrm{kA} / \mathrm{m} ; H=156 \mathrm{kA} / \mathrm{m} ; H=229 \mathrm{kA} / \mathrm{m} ; H=281 \mathrm{kA} / \mathrm{m}$.

Phil. Trans. R. Soc. A. 
To analyse more in details the effect of the applied magnetic field in the curves of inherent viscosity vs. Péclet number, it is convenient to plot separately the curves for samples Alg+Fe and $\mathrm{Alg}+\mathrm{Chi}+\mathrm{Fe}$, both in double logarithmic and double linear scales (Figure 5). For Alg+Fe sample (Figures 5a and 5b), a dramatic effect of the applied magnetic field can be observed. First, curves move to the right (higher Péclet number) as the magnetic field increases, because of the reduced mobility of particles that are forced to stack in structures aligned in the field direction. In addition, the magnetic field application provokes large increase of the inherent viscosity, as a result of the resistance of the particle structures to the flow provoked by shearing forces. As the magnetic field increases, inherent viscosity also increases, although a tendency of curves to overlap with the curve in the absence of applied magnetic field is observed as the Péclet number increases.

(a)

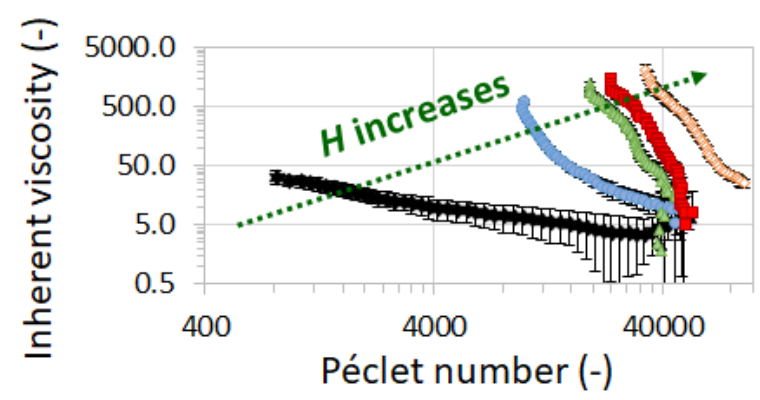

(c)

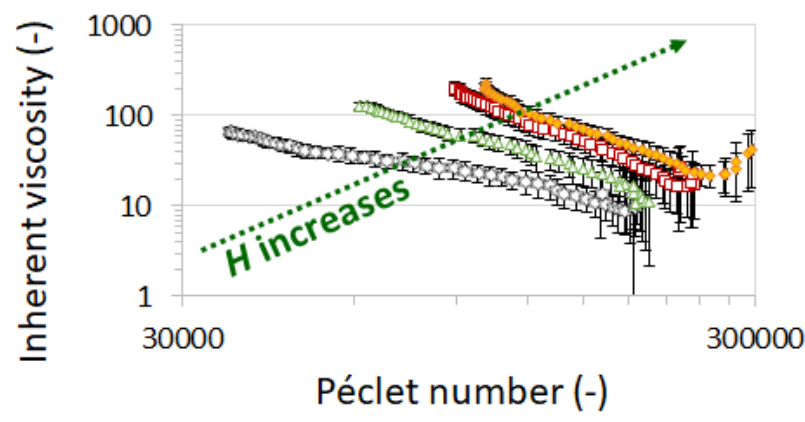

(b)

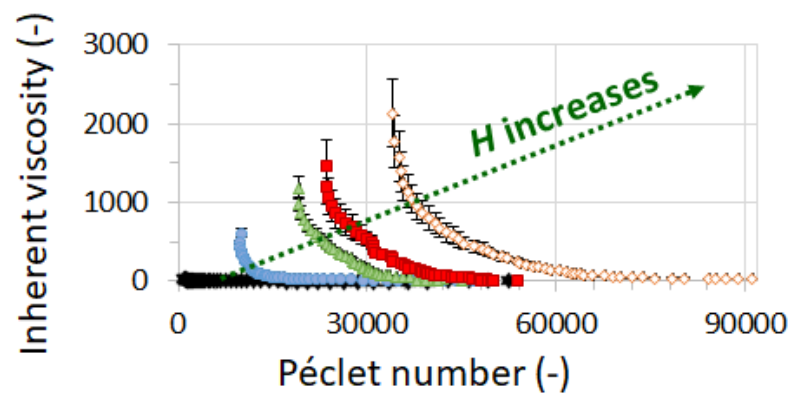

(d)

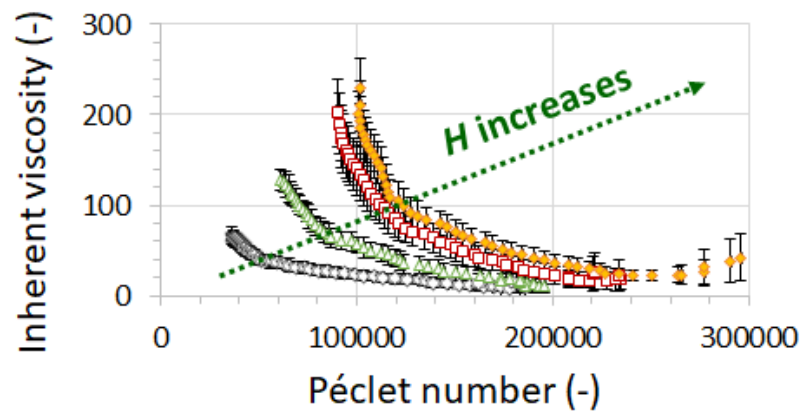

Figure 5. Inherent viscosity vs. Péclet number for different intensities of the applied magnetic field. (a) and (c) Double logarithmic scale; (b) and (d) Double linear scale. (a) and (b) Alg+Fe sample: suspension of iron particles at a concentration of 5 vol. \% in sodium alginate solution at 15 weight \%; (c) and (d) $\mathrm{Alg}+\mathrm{Chi}+\mathrm{Fe}$ sample: suspension of iron particles at a concentration of $5 \mathrm{vol} . \%$ in polymer solution containing 15 weight $\%$ of sodium alginate and 0.75 weight $\%$ of chitosan. In each graph, the applied magnetic field, $H$, increases from the curve most to the left to the curve most to the right accordingly to the following values: Alg+Fe: $H=0 \mathrm{kA} / \mathrm{m} ; H=73.5 \mathrm{kA} / \mathrm{m} ; H=156 \mathrm{kA} / \mathrm{m} ; H=229 \mathrm{kA} / \mathrm{m} ; H=281 \mathrm{kA} / \mathrm{m}$; Alg+Chi+Fe: $H=0 \mathrm{kA} / \mathrm{m} ; H=156 \mathrm{kA} / \mathrm{m} ; H=229 \mathrm{kA} / \mathrm{m} ; H=281 \mathrm{kA} / \mathrm{m}$.

These observations can be interpreted as it follows. At low Péclet number (low shear rate), magnetic forces dominate over thermal diffusion and hydrodynamic transport, justifying the exceptionally large values of inherent viscosity. As Péclet number increases, hydrodynamic transport is progressively overcoming magnetic forces, provoking a fracture and gradual disintegration of particle structures, resulting in a decrease of inherent viscosity. For the sample in the absence of applied magnetic field, ruling phenomenon is particle aggregates occurring by colloidal forces, mainly van der Waals attraction, in the absence of hydrodynamic forces, which is rapidly overcome by hydrodynamic transport as shearing forces are applied. At the highest values of Péclet number, both in the absence and presence of applied magnetic field, hydrodynamic transport dominates and thus, all curves seem to converge. In all cases, the very large values of Péclet number corroborate the negligible effect of thermal motion. For Alg+Chi+Fe sample, overall, the same trends are obtained, and a similar reasoning can be held. However, the effect of the applied magnetic field is smaller in this case, with respect to Alg+Fe sample, which is logical in view of the expected trapping of the particles in the gel-like polymer network. Thus, for this sample the increase of inherent viscosity with the applied magnetic field is smaller and their decrease as Péclet number grows is smaller too, evidencing a 
relatively smaller influence of both magnetic forces and particle aggregation, with respect to hydrodynamic transport.

(a)

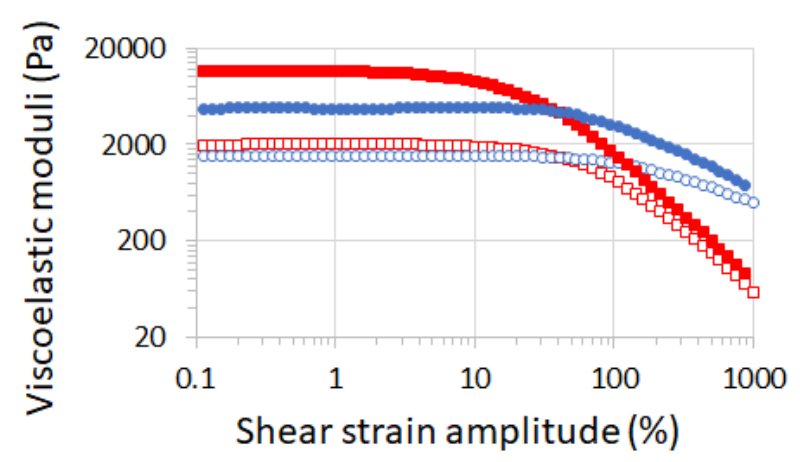

(c)

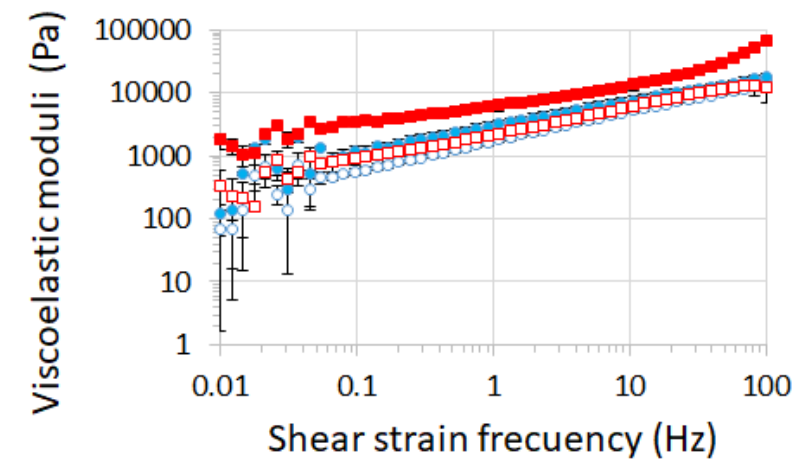

(b)

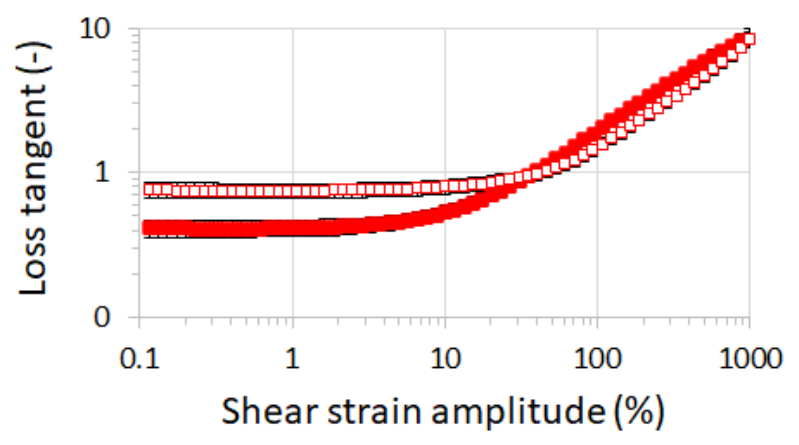

(d)

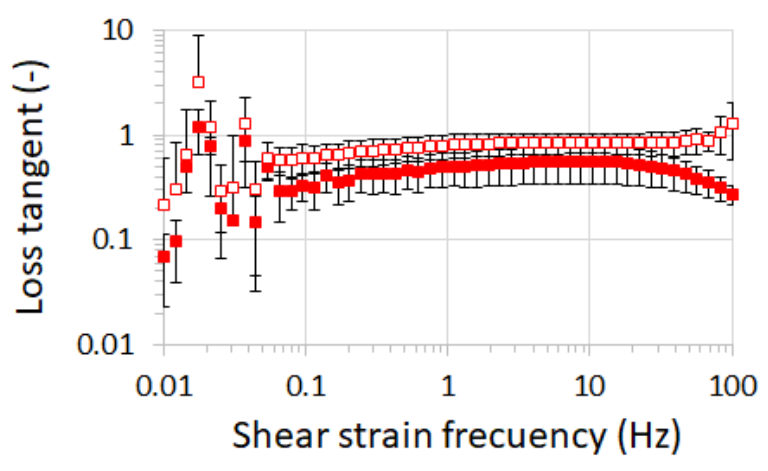

Figure 6. Viscoelastic moduli for Alg+Chi and Alg+Chi+Fe samples. (a) Amplitude sweeps: curves of viscoelastic moduli vs. shear strain amplitude for a fixed frequency of $1 \mathrm{~Hz}$. (b) Loss tangent vs. shear strain amplitude for a fixed frequency of $1 \mathrm{~Hz}$. (c) Frequency sweeps: curves of viscoelastic moduli vs. shear strain frequency for a fixed amplitude within the LVR. (d) Loss tangent vs. shear strain frequency for a fixed amplitude within the LVR. In (a) and (c) squares and circles represent respectively the values of the storage $\left(G^{\prime}\right)$ and loss $\left(G^{\prime \prime}\right)$ moduli. Open symbols are for Alg+Chi solution, whereas full symbols are for

Alg+Chi+Fe sample. Alg+Chi: polymer solution containing 15 weight \% of sodium alginate and 0.75 weight $\%$ of chitosan. Alg+Chi+Fe: suspension of iron particles at a concentration of 5 vol. \% in $\mathrm{Alg}+\mathrm{Chi}$ solution.

(c) Analysis of the dynamic regime of suspensions of iron particles in polymer solutions

In this subsection we will analyse first the effect of addition of iron particles at a concentration of 5 vol. $\%$ in the response of samples under oscillatory shear (Figure 6). As observed, the addition of iron particles to Alg+Chi solution results in an important increase of the viscoelastic moduli corresponding to the LVR (Figure 6a). What is more, the gel-like behaviour is enhanced, as loss tangent $\left(\tan \delta=\mathrm{G}^{\prime \prime} / \mathrm{G}^{\prime}\right.$ ) within the LVR decreases from 0.77 for Alg+Chi sample to 0.42 for Alg+Chi+Fe sample (at amplitude $0.1 \%$ ), indicating and strengthening of the gel like structure connected to the role of the particles within the polymer network (Figure 6b) - remark $1>\tan \delta>0.1$ is typical of weak gels [15]. Similar enhancements under the addition of magnetic particles to polymer hydrogels were reported in previous works [16],[18]. Despite this enhancement, the general trend of $G^{\prime}$ and $G^{\prime \prime}$ vs. shear strain amplitude remains similar, including the transition to the NLVR, and the point above which $G^{\prime \prime}$ overcomes $G^{\prime}$ that marks the transition from a gellike behaviour to a liquid-like behaviour. Also, note that in the NLVR curves for Alg+Chi solution and $\mathrm{Alg}+\mathrm{Chi}+\mathrm{Fe}$ sample tend to overlap (especially noticeable for $\tan \delta$ ), which corroborates that the important role of particles to give consistence to the gel network - once the network is broken, the role of particles in the rheological properties of the sample is irrelevant. For Alg samples, as we reported in a previous work 
[11], the addition of iron particles resulted in slight increase of $G^{\prime}$ and $G^{\prime \prime}$, without affecting to the liquidlike behaviour of these samples.

The analysis of the dependence of the viscoelastic moduli with frequency of oscillatory shear (mechanical spectra, Figure $6 \mathrm{c}$ and $6 \mathrm{~d}$ ) confirmed the increase of both $\mathrm{G}^{\prime}$ and $\mathrm{G}^{\prime \prime}$ when the iron particles were added to the Alg+Chi solution, enhancing the gel-like behaviour for the complete range of frequencies under study. Regarding the trends exhibited by viscoelastic moduli with frequency, as observed both $G^{\prime}$ and $G^{\prime \prime}$ increase mildly with the latter (almost linearly in the double logarithmic scale), which is typical of weak gels [15]. What is more, as evidenced by plots of $\tan \delta$ vs. frequency, the former tends to increase slightly with the latter, although a final decrease is achieved for sample $\mathrm{Alg}+\mathrm{Chi}+\mathrm{Fe}-$ noisy points in $\tan \delta$ at low frequencies should be connected to error in the determination of $\mathrm{G}^{\prime \prime}$. For Alg+Chi solution, the value of $\tan \delta=1$ (transition to liquid-like behaviour) is achieved at the higher frequencies under study.

(a)

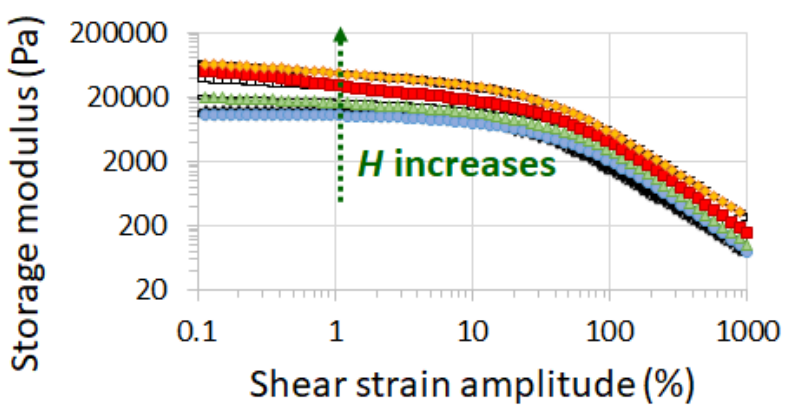

(c)

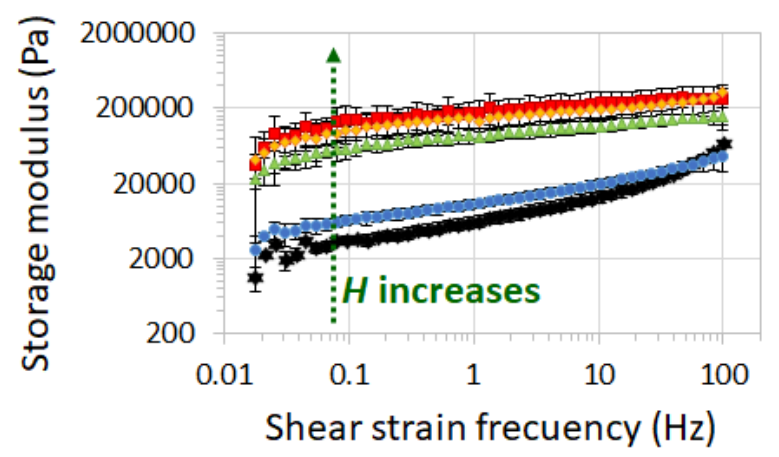

(b)

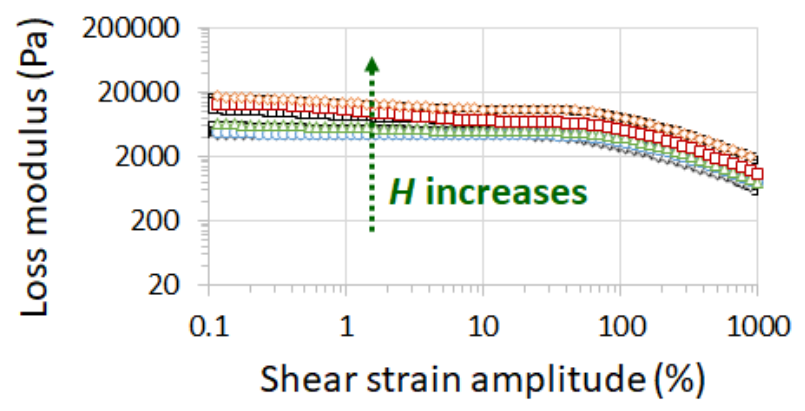

(d)

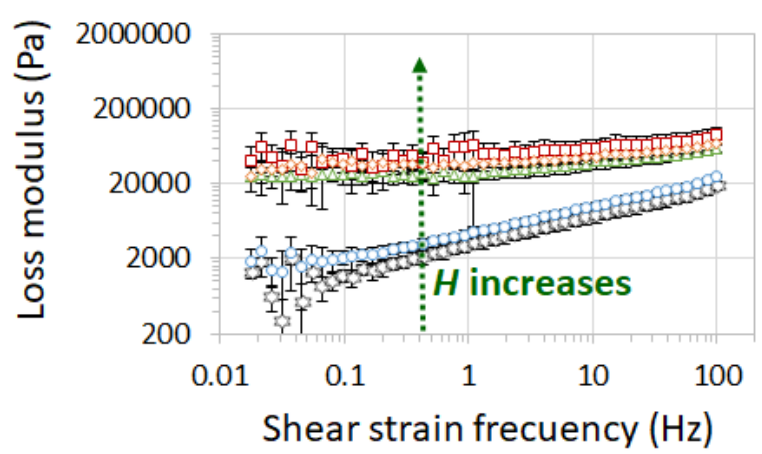

Figure 7. Viscoelastic moduli for $\mathrm{Alg}+\mathrm{Chi}+\mathrm{Fe}$ samples under different applied magnetic fields. (a) and (b) Amplitude sweeps: curves of viscoelastic moduli vs. shear strain amplitude for a fixed frequency of 1

Hz. (c) and (d) Frequency sweeps: curves of viscoelastic moduli vs. shear strain frequency for a fixed amplitude within the LVR. In each graph, the applied magnetic field, $H$, increases from the curve most to the bottom to the curve most to the top accordingly to the following values: $H=0 \mathrm{kA} / \mathrm{m} ; H=73.5 \mathrm{kA} / \mathrm{m}$; $H=156 \mathrm{kA} / \mathrm{m} ; H=229 \mathrm{kA} / \mathrm{m} ; H=281 \mathrm{kA} / \mathrm{m}$. Note that the same axis scales are used in parts (a) and (b) and in parts (c) and (d).

Let us now analyse the role the applied magnetic field on the response to oscillatory shear of the Alg+Chi+Fe sample (Figures 7 and 8). As can be observed in Figure 7, the application of a magnetic field does not affect the general trends of the viscoelastic moduli with strain amplitude and strain frequency, with respect to the absence of applied magnetic field. The role of the applied magnetic field is reduced to the progressive enhancement of $G^{\prime}$ and $G^{\prime \prime}$ as the field is increased. This enhancement reached almost one order of magnitude for the highest field, something that is extraordinary for a $5 \mathrm{vol} . \%$ of iron particles. For comparison, note that a similar enhancement was reached for alginate ferrogels crosslinked by calcium ions at a similar concentration of iron particles [10]. In another work, Mitsumata et al. [19] reported a two-orders of magnitude increase for the storage modulus for magnetic hydrogels consisting of carrageenan and carbonyl iron particles at $30 \mathrm{vol} . \%$. These enhancements of storage modulus for ferrogels were termed as huge or giant, since most magnetic hydrogels exhibited minor changes of only a few percent under a magnetic field [20],[21]. These large increases of the viscoelastic moduli can only be explained by the field- 
induced migration of particles in weak polymer networks [10]. For strong polymer networks, elasticity strongly hinders migration of the magnetic particles, preventing from large changes of the rheological properties under a magnetic field.

From Figure 8, an overall tendency of the mean values of loss tangent to diminish with the intensity of the field is noticeable, especially within the LVR. This is justified by the strengthening of the microstructure of the suspensions of iron particles. Note, however, than even at the highest magnetic field, the values of loss tangent within the LVR corresponds to weak gels, $1>\tan \delta>0.1$ [15].
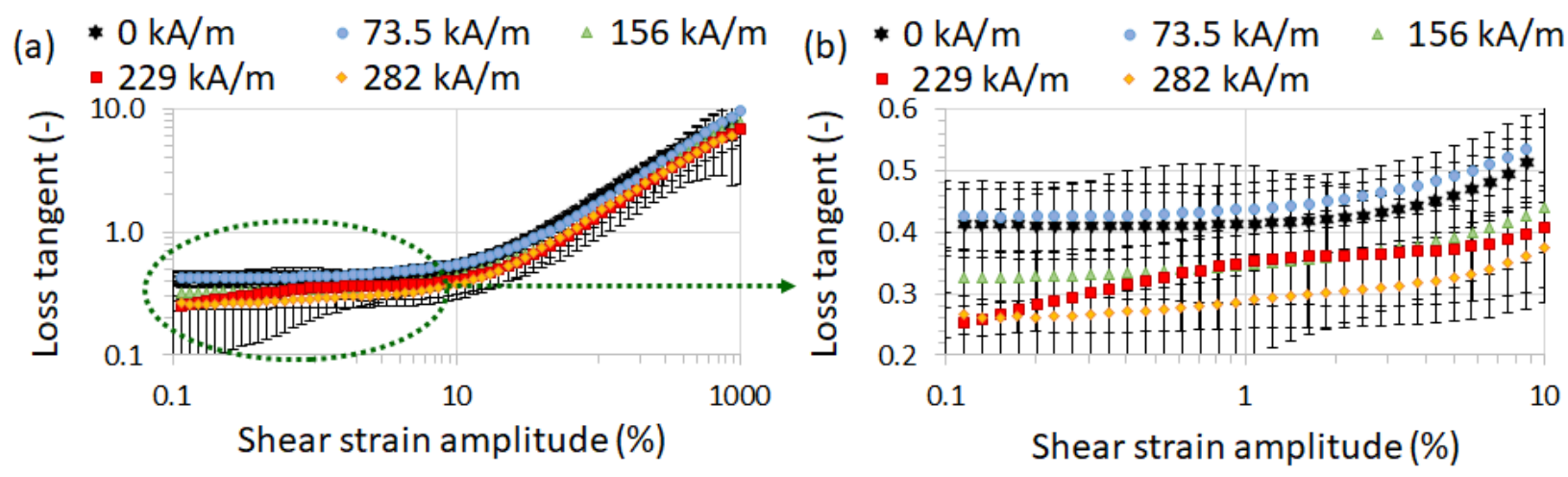

Figure 8. Loss tangent vs. shear strain amplitude for $\mathrm{Alg}+\mathrm{Chi}+\mathrm{Fe}$ samples under different applied magnetic fields. (a) Double logarithmic scale; (b) Zoom of data corresponding to the linear viscoelastic region, with linear scale in the vertical axis and logarithmic scale in the horizontal axis. The values of the applied magnetic field strengths $(H, \mathrm{kA} / \mathrm{m})$ are indicated.

The use of dimensionless numbers to analyse the response of samples to oscillatory shear is not common. Nevertheless, we could adapt the concept of Péclet number and inherent viscosity - equations (1) and (2) to oscillatory results. For this aim, instead of the Péclet number, defined as in equation (1), we can define, $\mathrm{Pe}^{\prime}$, by substituting the shear stress of steady state measurements, $\sigma$, by the shear stress amplitude, $\sigma_{0}$, of the oscillatory shear:

$$
P e^{\prime} \equiv \frac{\sigma_{0} a^{3}}{k T} .
$$

Similarly, we can define a dimensionless modulus, by adaptation of equation (2) as it follows:

$$
G_{i n h} \equiv \frac{G-G_{0}}{\phi G_{0}},
$$

where $G$ can be either the storage or the loss modulus. Similarly to the case of steady-state studies, this analysis using inherent moduli and Péclet number in oscillometry allows getting a complete view of the differences between samples and experimental conditions in a single graph (Figure 9). As observed, representation of the inherent modulus in the vertical axis provides information of the normalized differences in modulus for the different samples and experimental conditions. In addition, using Péclet number in the horizontal axis gives a much better idea than the shear strain amplitude (compare Figure 9 with Figure 7) of the strength of the sample and allows differentiating better between samples. In the particular analysis of the current work, from Figure 9 we see that the effect of the magnetic field is stronger for Alg+Fe sample than for Alg+Chi+Fe sample. Furthermore, it is evident that as hydrodynamic forces increase (higher Péclet number), the role of magnetic forces diminishes. Also, with the increase of Péclet number, and the consequent increase of transport phenomena, large particle aggregates are broken, resulting in smaller differences between samples. This effect is more gradual for $\mathrm{Alg}+\mathrm{Chi}+\mathrm{Fe}$ samples, since for them the role of magnetic field is smaller and, what is more, the entanglement between polymer networks seems to remain even at very large Péclet number, despite the dominant role played by the hydrodynamic forces in the transport phenomena. 
(a)

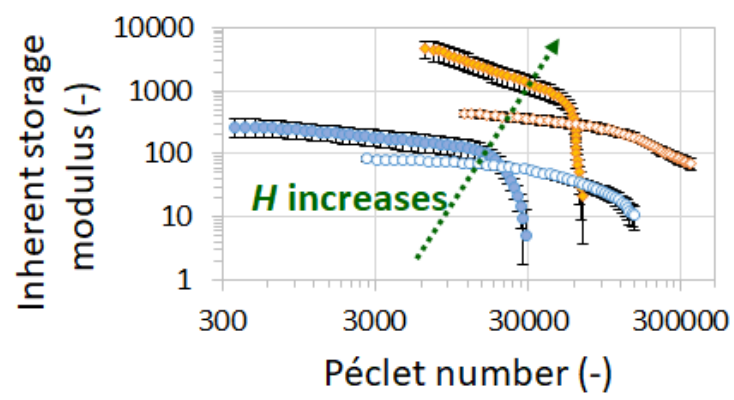

(b)

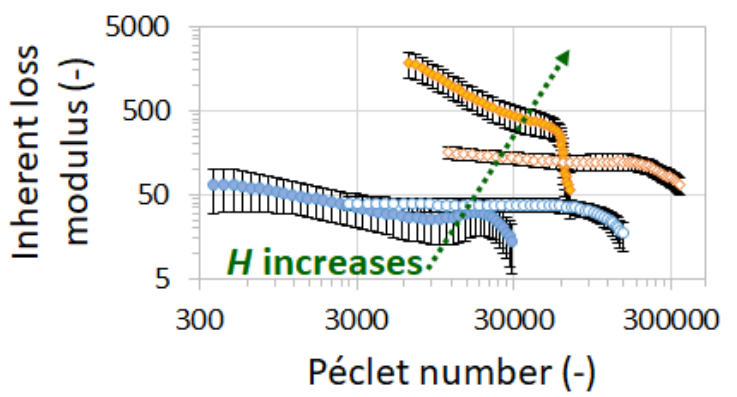

Figure 9. Inherent viscoelastic moduli vs. Péclet number under different applied magnetic fields. Full symbols are for Alg+Fe sample, whereas open symbols are for Alg+Chi+Fe sample. Alg+Fe: suspension of iron particles at a concentration of 5 vol. \% in sodium alginate solution at 15 weight \%; $\mathrm{Alg}+\mathrm{Chi}+\mathrm{Fe}$ : suspension of iron particles at a concentration of $5 \mathrm{vol} . \%$ in polymer solution containing 15 weight $\%$ of sodium alginate and 0.75 weight $\%$ of chitosan. In each graph and for each sample, the applied magnetic field, $H$, increases from the curve most to the bottom to the curve most to the top accordingly to the following two values: $H=73.5 \mathrm{kA} / \mathrm{m} ; H=281 \mathrm{kA} / \mathrm{m}$. Data in this figure are for shear strain amplitudes in the range $1 \%-500 \%$.

(d) Self-healing analysis

In this final subsection we indirectly analyse the effect of a large shear rate on the microstructure of the Alg+Chi+Fe sample (Figure 10). As observed, before the application of the large shear rate, the sample demonstrates very large values of the storage and loss moduli, as well as an evident gel-like behaviour, characterized by values of the storage modulus larger than these of the loss modulus. Immediately after the large shear rate application (see data around $200 \mathrm{~s}$ ), the situation is opposite, with a large decrease (minimum) of the viscoelastic moduli (more than two orders of magnitude) and the loss modulus larger than the storage modulus, i.e., liquid-like behaviour. This is due to the breakage of the polymer network. After the minimum in viscoelastic moduli, both storage and loss moduli progressively increase, with an eventual crossover of $G^{\prime}$ and $G^{\prime \prime}$, returning to the gel-like character $\left(G^{\prime}>G^{\prime \prime}\right)$ at $t \approx 218$ s. Nevertheless, despite the progressive increase in viscoelastic moduli, the values prior to application of large shear rate are not achieved during the duration of the experiment, with the maximum regained values of storage and loss modulus being more than one order of magnitude smaller than those showed before destruction of the internal structure. This self-healing behaviour contrasts with this of gel-like samples based on entanglement of polymers (without electrostatic interaction between polymer in solution), for which a recovery of more than $70 \%$ of the values prior to breakage was reported in our previous work [11]. In the case of the present work, rebuilding of electrostatic bonding between alginate and chitosan, although reversible in view of the progressive self-healing manifested in Figure 10, presumably requires of long time to be completed. Note, however, that a gel-like behaviour is rapidly recovered, which represents an interesting feature with respect to covalent hydrogels that usually lack of self-healing mechanisms [22]. 


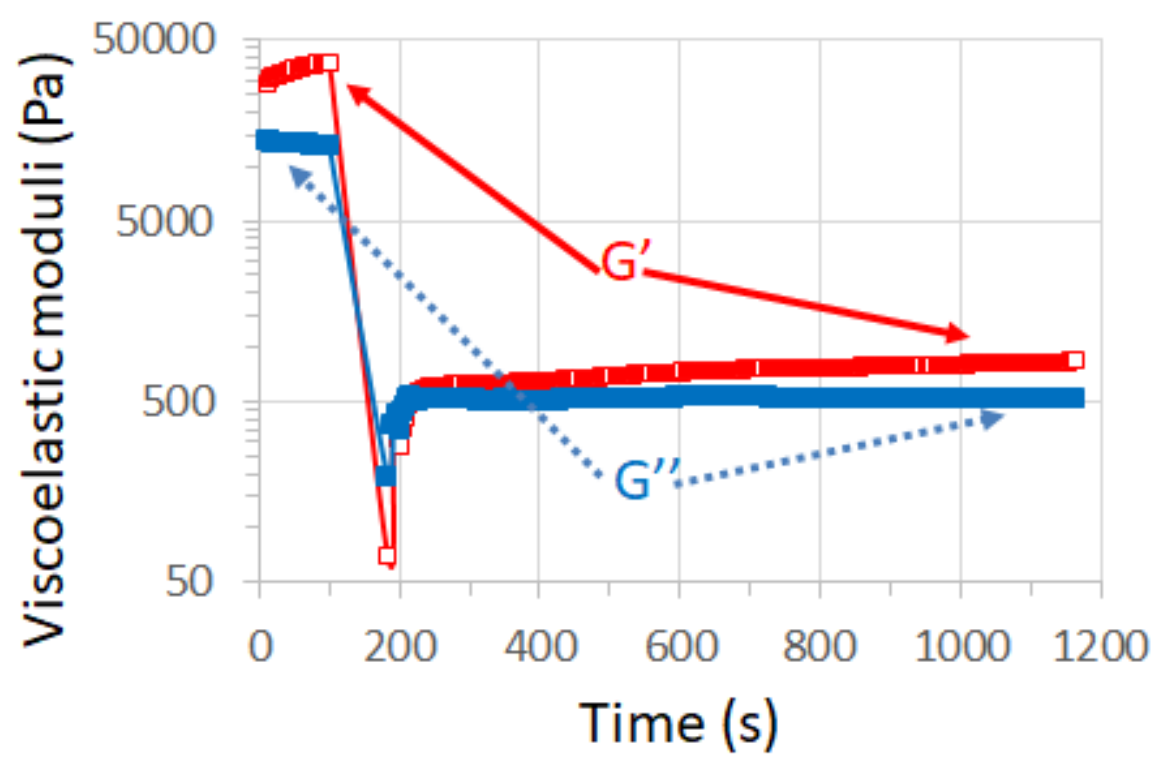

Figure 10. Self-healing behaviour of Alg+Chi+Fe sample: suspension of iron particles at a concentration of 5 vol. \% in polymer solution containing 15 weight \% of sodium alginate and 0.75 weight $\%$ of chitosan. Symbols represent the values of the viscoelastic moduli resulting from an oscillatory shear strain of $0.1 \%$ of strain amplitude and $1 \mathrm{~Hz}$ of frequency. During the time interval 100-160 s viscoelastic moduli were not recorded, an instead, the sample was subjected to a shear rate of $1000 \mathrm{~s}^{-1}$. Open squares (red) represent the storage modulus $\left(G^{\prime}\right)$ and full symbols (blue) represent the loss modulus $\left(G^{\prime \prime}\right)$. Lines are guides for the eye.

The curves represent a single test, although we performed three different repetitions with different aliquots obtaining similar results.

\section{Conclusions}

Contrarily to liquid-like solutions of alginate polymers, mixed solutions of anionic alginate polymers and cationic chitosan polymers under mildly acidic conditions show gel-like rheological properties, very likely due to the electrostatic attraction between oppositely charged polymer chains. Addition of magnetic iron particles to these mixed solutions results in magneto-polymer composites that demonstrate responsiveness to the magnetic field action. The use of dimensionless numbers allows a fair comparison between samples and experimental conditions, as well as analysis of the relevance of different phenomena and dominant forces on the flow of these magneto-polymer composites. The large values of Péclet number demonstrate that thermal diffusion is negligible, whereas the strong shear thinning behaviour demonstrated by samples containing particles must be connected to the breakage of particle clusters in the absence of applied magnetic field. When a magnetic field is applied, magnetic forces of attraction between particles clearly dominate over thermal diffusion, which results in strong changes in the rheological properties under increasing values of the applied field. At large values of Péclet number, the transport induced by bulk hydrodynamic forces clearly dominates and the inherent rheological parameters tend to overlap for the different values of the applied magnetic field.

\section{Funding Statement}

W.R.S.-F. acknowledges financial support by the UTE University of Ecuador through paid licenses for trips and stays from Ecuador to Spain.

J.D.G.D. received support from the Ministerio de Economía, Industria y Competitividad, MINECO, and Agencia Estatal de Investigación, AEI, Spain, cofounded by Fondo Europeo de Desarrollo Regional, FEDER, European Union, project FIS2017-85954-R. 
M.T.L.-L. received support from the Ministerio de Economía, Industria y Competitividad, MINECO, and Agencia Estatal de Investigación, AEI, Spain, cofounded by Fondo Europeo de Desarrollo Regional, FEDER, European Union, project FIS2017-85954-R.

\section{Data Accessibility}

The datasets supporting this article have been uploaded as Supplementary Material.

\section{Competing Interests}

We declare we have no competing interests.

\section{Authors' Contributions}

W.R.S.-F.: substantial contributions to acquisition of data; drafting the article; final approval of the version to be published.

J.D.G.D.: substantial contributions to conception and design, and analysis and interpretation of data; revising the article critically for important intellectual content; final approval of the version to be published. M.T.L.-L.: substantial contributions to conception and design, analysis and interpretation of data; drafting the article; final approval of the version to be published.

\section{References}

1. Chai Q, Jiao Y, Yu X. 2017 Hydrogels for Biomedical Applications: Their Characteristics and the Mechanisms behind Them. Gels (Basel, Switzerland) 3, 6. (doi:10.3390/gels3010006)

2. Caló E, Khutoryanskiy V V. 2015 Biomedical applications of hydrogels: A review of patents and commercial products. Eur. Polym. J. 65, 252-267. (doi:10.1016/J.EURPOLYMJ.2014.11.024)

3. Ahmed EM. 2015 Hydrogel: Preparation, characterization, and applications: A review. J. Adv. Res. 6, 105-121. (doi:10.1016/j.jare.2013.07.006)

4. Sudhakar YN, Selvakumar M, Bhat DK. 2018 An introduction of Biopolymer Electrolytes. In Biopolymer Electrolytes, pp. 1-34. Elsevier. (doi:10.1016/b978-0-12-813447-4.00001-7)

5. Almdal K, Dyre J, Hvidt S, Kramer O. 1993 Towards a phenomenological definition of the term 'gel'. Polym. Gels Networks 1, 5-17. (doi:10.1016/0966-7822(93)90020-I)

6. Clark AH, Ross-Murphy SB. 2005 Structural and mechanical properties of biopolymer gels. In Biopolymers, pp. 57-192. Springer-Verlag. (doi:10.1007/bfb0023332)

7. Zhao F, Yao D, Guo R, Deng L, Dong A, Zhang J. 2015 Composites of Polymer Hydrogels and Nanoparticulate Systems for Biomedical and Pharmaceutical Applications. Nanomaterials 5, 2054 2130. (doi:10.3390/nano5042054)

8. Lopez-Lopez MT, Durán JDG, Iskakova LY, Zubarev AY. 2016 Mechanics of Magnetopolymer Composites: A Review. J. Nanofluids 5, 479-495. (doi:10.1166/jon.2016.1233)

9. Goudu SR, Yasa IC, Hu X, Ceylan H, Hu W, Sitti M. 2020 Biodegradable Untethered Magnetic Hydrogel Milli-Grippers. Adv. Funct. Mater. 30, 2004975. (doi:10.1002/adfm.202004975)

10. Gila-Vilchez C, Duran JDG, Gonzalez-Caballero F, Zubarev A, Lopez-Lopez MT. 2019 Magnetorheology of alginate ferrogels. Smart Mater. Struct. 28, 035018. (doi:10.1088/1361665X/aafeac)

11. Suarez-Fernandez WR, Scionti G, Duran JDGG, Zubarev AY, Lopez-Lopez MT. 2020 Role of particle clusters on the rheology of magneto-polymer fluids and gels. Philos. Trans. R. Soc. A Math. Phys. Eng. Sci. 378, 20190254. (doi:10.1098/rsta.2019.0254)

12. Wagner NJ, Brady JF. 2009 Shear thickening in colloidal dispersions. Phys. Today 62, 27-32. (doi:10.1063/1.3248476)

13. Bossis G, Volkova O, Lacis S, Meunier A. 2002 Magnetorheology: Fluids, Structures and Rheology BT - Ferrofluids: Magnetically Controllable Fluids and Their Applications. In (ed S Odenbach), pp. 202-230. Berlin, Heidelberg: Springer Berlin Heidelberg. (doi:10.1007/3-540-45646-5_11)

14. Nurunnabi M, Revuri V, Huh KM, Lee Y kyu. 2017 Polysaccharide based nano/microformulation: An effective and versatile oral drug delivery system. In Nanostructures for Oral Medicine, pp. 409433. Elsevier Inc. (doi:10.1016/B978-0-323-47720-8.00015-8)

15. Borzacchiello A, Della Sala F, Ambrosio LA. 2017 Rheometry of polymeric biomaterials. In 
Characterization of Polymeric Biomaterials, pp. 233-253. Elsevier. (doi:10.1016/B978-0-08-1007372.00010-8)

16. Gila-Vilchez C, Bonhome Espinosa A, Kuzhir P, Zubarev A, D. G. Duran J, López-López M. 2018 Rheology of magnetic alginate hydrogels. J. Rheol. (N. Y. N. Y). 62, 1083-1096.

(doi:10.1122/1.5028137)

17. López-López MT, Kuzhir P, Bossis G, Mingalyov P. 2008 Preparation of well-dispersed magnetorheological fluids and effect of dispersion on their magnetorheological properties. Rheol. Acta 47, 787-796. (doi:10.1007/s00397-008-0271-6)

18. Bonhome-Espinosa AB, Campos F, Rodriguez IA, Carriel V, Marins JA, Zubarev A, Duran JDG, Lopez-Lopez MT. 2017 Effect of particle concentration on the microstructural and macromechanical properties of biocompatible magnetic hydrogels. Soft Matter 13, 2928-2941. (doi:10.1039/c7sm00388a)

19. Mitsumata T, Honda A, Kanazawa H, Kawai M. 2012 Magnetically Tunable Elasticity for Magnetic Hydrogels Consisting of Carrageenan and Carbonyl Iron Particles. J. Phys. Chem. B 116, 1234112348. (doi:10.1021/jp3049372)

20. Lopez-Lopez MT, Scionti G, Oliveira AC, Duran JDG, Campos A, Alaminos M, Rodriguez IA. 2015 Generation and Characterization of Novel Magnetic Field-Responsive Biomaterials. PLoS One 10, e0133878. (doi:10.1371/journal.pone.0133878)

21. Abrougui MM, Srasra E, Lopez-Lopez MT, Duran JDG. 2020 Rheology of magnetic colloids containing clusters of particle platelets and polymer nanofibres. Philos. Trans. R. Soc. A Math. Phys. Eng. Sci. 378, 20190255. (doi:10.1098/rsta.2019.0255)

22. Zhang L, Tian M, Wu J. 2016 Hydrogels with Self-Healing Attribute. In Emerging Concepts in Analysis and Applications of Hydrogels, InTech. (doi:10.5772/64138) 